\title{
LA ENSEÑANZA DE LA RELACIÓN MÉDICO-PACIENTE CON PODER TERAPÉUTICO: ENFOCADO CON LA TEORÍA FUNDAMENTADA CONSTRUCTIVISTA
}

\section{TEACHING THE DOCTOR-PATIENT RELATIONSHIP WITH THERAPEUTIC POWER: FOCUSED WITH CONSTRUCTIVIST GROUNDED THEORY}

Ismael Ramírez-Villaseñor*

Investigación Psicológica, Instituto Tecnológico y de Estudios Superiores de Occidente, Guadalajara, Jalisco, México

\begin{abstract}
RESUMEN: Antecedentes: La educación médica formal ha dejado fuera la idea de efecto terapéutico/dañino en la relación médico-paciente a pesar de los trabajos de Michael Balint y los acumulados desde 1960 a la fecha que lo prueban. La formación en ese campo subjetivo es omitida en el currículo médico. Objetivo: Explorar lo que piensan profesores de medicina familiar respecto a la posible enseñanza de los elementos terapéuticos de la relación médico-paciente. Métodos: Entrevistas semiestructuradas a dos profesores experimentados de medicina familiar, usando el método cualitativo de investigación y la teoría fundamentada como medio de análisis de los datos. Resultados: Los profesores describieron elementos y barreras implicados en la enseñanza de la relación médico-paciente con poder terapéutico. Conclusión: Son elementos terapéuticos la capacidad empática y el desarrollo de autoconciencia; un medio de desarrollo es la reflexión sobre los casos de pacientes «difíciles». El ambiente hospitalario podría ser dañino para el desarrollo empático. La residencia de medicina familiar en México podría estar fallando para formar residentes con capacidades para formar relaciones médico-paciente terapéuticas.
\end{abstract}

Palabras clave: Relación médico-paciente. Autoconciencia. Empatía. Educación médica. Compasión. Teoría fundamentada constructivista.
ABSTRACT: Background: Formal medical education has left out the idea of therapeutic / harmful effect in the doctor-patient relationship despite the works of Michael Balint and those accumulated from 1960 to date. Training in this subjective field is omitted in the medical curriculum. Objective: To explore what family medicine teachers think about the possible teaching of the therapeutic elements of the doctor-patient relationship. Methods: Semi-structured interviews with 2 experienced family medicine teachers, using the qualitative research method and grounded theory as a means of data analysis. Results: The teachers described elements and barriers involved in teaching the doctor-patient relationship with therapeutic power. Conclusion: The empathic capacity and the development of self-awareness are therapeutic elements; a way of development is reflection on "difficult" patients. The hospital environment could be detrimental to empathic development. The family medicine residency in Mexico could be failing to train residents with skills to form therapeutic doctor-patient relationships.

Keywords: Physician-Patient relation. Self-perception. Empathy. Medical education. Compassion. Grounded theory.
Correspondencia:

*Ismael Ramírez-Villaseñor

E-mail: ismaelravi@gmail.com
Fecha de recepción: 24-08-2021

Fecha de aceptación: 18-12-2021
Disponible en internet: 24-02-2022

Rev Mex Med Fam. 2022;9:20-30 DOI: 10.24875/RMF.21000082

2007-9710 / @ 2021 Federación Mexicana de Especialistas y Residentes en Medicina Familiar. Publicado por Permanyer. Este es un artículo open access bajo la licencia CC BY-NC-ND (http://creativecommons.org/licenses/by-nc-nd/4.0/). 


\section{ANTECEDENTES}

Los trabajos de Michael y Enid Balint con médicos generales de las décadas de 1950 a 1970 demostraron que el médico general es en sí mismo(a) el mejor recurso terapéutico que podría ofrecer a sus pacientes. Llamaron a este complejo concepto médico-medicamento $^{1}$; usaron la metáfora farmacológica para explicar que la personalidad de cada médico es como los fármacos, no tienen los mismos efectos en todos los pacientes, ocurren alergias y otros efectos adversos; pueden ocurrir intoxicaciones, producir dependencia, pero bien indicados y dosificados son de indudable ayuda. La tarea personal de cada médico general es entonces desarrollar autoconocimiento de nuestro perfil terapéutico para prescribirnos adecuadamente en cada caso ${ }^{1}$. En el posterior desarrollo de la disciplina científico-académica que dio lugar al renacimiento de la medicina general en su forma moderna bajo el nombre de medicina familiar, se desarrolló la idea de los Balint en el concepto de relación médico-paciente con poder terapéutico ${ }^{2,3}$ y que su componente principal es una postura mental que combina afecto y caridad, dos de los componentes del amor en el concepto griego clásico de agape $e^{2}$. Desde la psiquiatría la misma idea se ha conceptualizado como la unión de la ecuanimidad y la compasión, o ecuanimidad-compasiva. La idea de ecuanimidad compasiva se encuentra por primera vez en Adler, pero no describe con precisión su constructo, solo sus efectos clínicos en la fisiología de psiquiatras y pacientes ${ }^{3}$. Estos conceptos incluidos por completo en la esfera de la subjetividad no han encontrado espacio formal en la educación médica dominada por el paradigma neopositivista ${ }^{4,5}$ a pesar del importante número de pruebas acumuladas de que la relación médico-paciente influye poderosamente en los desenlaces clínicos ${ }^{6-8}$.

El presente estudio exploratorio sustentado en la teoría constructivista es parte de un proyecto doctoral que pone a prueba un método complejo de educación emocional en el contexto clínico construido en seis años de experiencias con estudiantes y médicos ${ }^{9,10}$. Y que está avanzando en la enseñanza aprendizaje de la configuración subjetiva compleja denominada ecuanimidad compasiva, consciente de que la compasión sin equilibrio ecuánime puede ocasionar «fatiga», es decir: "sufrimiento emocional causado por la expresión prolongada y repetida de compasión o empatía» ${ }^{11}$. Por tanto, se propone la enseñanza de la compasión unida a la ecuanimidad. Esta se entiende como la postura de mente clara que no es perturbada por emociones y que permite la imparcialidad de juicio ${ }^{12}$. A su vez, la compasión se define como la intensificación de la empatía afectiva en el contexto del sufrimiento ${ }^{13}$. Entonces, se conceptúa la ecuanimidad compasiva como una configuración subjetiva ${ }^{14}$, un sistema autopoiético, que emerge de la interacción de emociones y procesos simbólicos, modulados por procesos de autoconocimiento. Incluye la capacidad de experimentar el sufrimiento del otro en la proporción necesaria y modulada por la ecuanimidad en los diversos contextos de la relación médico-paciente, así como el genuino deseo de aliviar el sufrimiento del otro con las acciones necesarias para ello ${ }^{15}$.

El sustento metodológico puede ser mejor comprendido a partir de la tabla 1 . Aunque el objetivo de este manuscrito no es discutir los consensos actuales del espectro de las teorías fundamentadas, la tabla 1 podría ser una útil orientación.

\section{PLANTEAMIENTO DEL PROBLEMA}

Aceptando que el médico general por su característica relación estrecha y a plazo indefinido con sus pacientes es capaz de ejercer cierto poder terapéutico (o dañino) en los resultados clínicos, queda por responder la pregunta de si ese poder terapéutico puede ser reconocido por los profesores de medicina y en especial los profesores de las residencias de medicina familiar y si ellos (as) creen que puede ser enseñado en el contexto clínico mexicano, no en la teoría. 
Tabla 1. Comparación de algunas características de la teoría fundamentada objetivista y la teoría fundamentada constructivista $^{16}$

\begin{tabular}{|c|c|}
\hline Teoría fundamentada objetivista & Teoría fundamentada constructivista (interpretativa) \\
\hline $\begin{array}{l}\text { La realidad externa es una sola y parte de un mundo } \\
\text { cognoscible }\end{array}$ & $\begin{array}{l}\text { Las realidades son múltiples, como plantean el interaccionismo simbólico y el } \\
\text { construccionismo social }\end{array}$ \\
\hline $\begin{array}{l}\text { Considera los datos como reales, objetivos en sí } \\
\text { mismos, encontrados por el investigador y que } \\
\text { descubre teoría para ellos }\end{array}$ & $\begin{array}{l}\text { Los datos se crean por las experiencias compartidas de investigador y partici- } \\
\text { pantes. La teoría que resulta es una interpretación del investigador }\end{array}$ \\
\hline $\begin{array}{l}\text { Trata a las categorías emergentes como resultados } \\
\text { automáticos }\end{array}$ & Los participantes construyen significados y acciones en situaciones específicas \\
\hline $\begin{array}{l}\text { No deja clara la postura interpretativa del investi- } \\
\text { gador }\end{array}$ & $\begin{array}{l}\text { Conducir y escribir una investigación no es un acto neutral, los investigadores } \\
\text { que llegan al campo con ideas similares y pueden producir teorías diferentes }\end{array}$ \\
\hline $\begin{array}{l}\text { Borra el contenido social, la influencia del inves- } \\
\text { tigador y las interacciones entre investigador y } \\
\text { participantes }\end{array}$ & $\begin{array}{l}\text { Los significados pueden representar ideologías, relaciones de poder, con- } \\
\text { venciones sociales, discursos, y ser parte de una realidad escondida mucho } \\
\text { mayor. Puede haber agudas discrepancias entre investigador y participantes }\end{array}$ \\
\hline $\begin{array}{l}\text { Enfatiza el desarrollo de categorías teóricas y las } \\
\text { trata como variables objetivas. No reconoce la } \\
\text { subjetividad en el proceso de investigación }\end{array}$ & Mucho de lo que ocurre en la investigación es tácito y silente, intersubjetivo \\
\hline $\begin{array}{l}\text { Busca lograr parsimonia y poder explicativo en } \\
\text { espectro } \\
\text { Ve la reflexión como una fuente posible de datos }\end{array}$ & $\begin{array}{l}\text { Busca comprensión interpretativa situada en tiempo, espacio, acción e } \\
\text { interacciones. La generalización es condicional } \\
\text { La reflexión es un compromiso durante todo el proceso, para limitar la repro- } \\
\text { ducción de ideologías y convencionalismos }\end{array}$ \\
\hline
\end{tabular}

- Pregunta de investigación 1: ¿Cuáles serían los elementos terapéuticos de la relación médico-paciente?

- Pregunta de investigación 2: ¿Es posible enseñar a los estudiantes/residentes los elementos terapéuticos esbozados aquí?

\section{Método}

El estudio utiliza el método cualitativo dentro del marco epistemológico constructivista y el análisis de los datos se hace conforme a la teoría fundamentada (Grounded theory) surgida en la década de 1960 en la sociología médica ${ }^{17}$. Es importante precisar que el análisis cualitativo se enfoca en los significados e interpretaciones que los investigados ya han interpretado de su realidad, y que los investigadores buscamos comprender trabajando con procesos y contenidos como en el caso de las entrevistas aquí descritas ${ }^{18}$. En este punto, la medicina familiar académica por su naturaleza interdisciplinaria está en excelente posición intelectual para comprender el estado actual de las grandes controversias que se discuten hoy en día respecto a los paradigmas científicos dentro de la investigación cualitativa. Sin intentar resolverlos, se ofrece una explicación concisa del estado de la discusión en la tabla 2.

\section{Participantes}

Los participantes fueron dos profesores de medicina familiar miembros del Colegio Jalisciense de Medicina Familiar A.C. organismo dedicado a la educación médica continua desde hace 27 años. Uno de los participantes tiene 30 años como docente en este campo y la otra participante tiene 10 años. Ambos enseñan en escuelas de medicina de universidades privadas de Guadalajara. Ambos son conocidos por el investigador por el trabajo conjunto de más de una década en la educación médica continua en Jalisco; el investigador, por tanto, forma parte del campo investigado, con sus ventajas y desventajas. La invitación a participar en el estudio se hizo de manera personal por medio de 


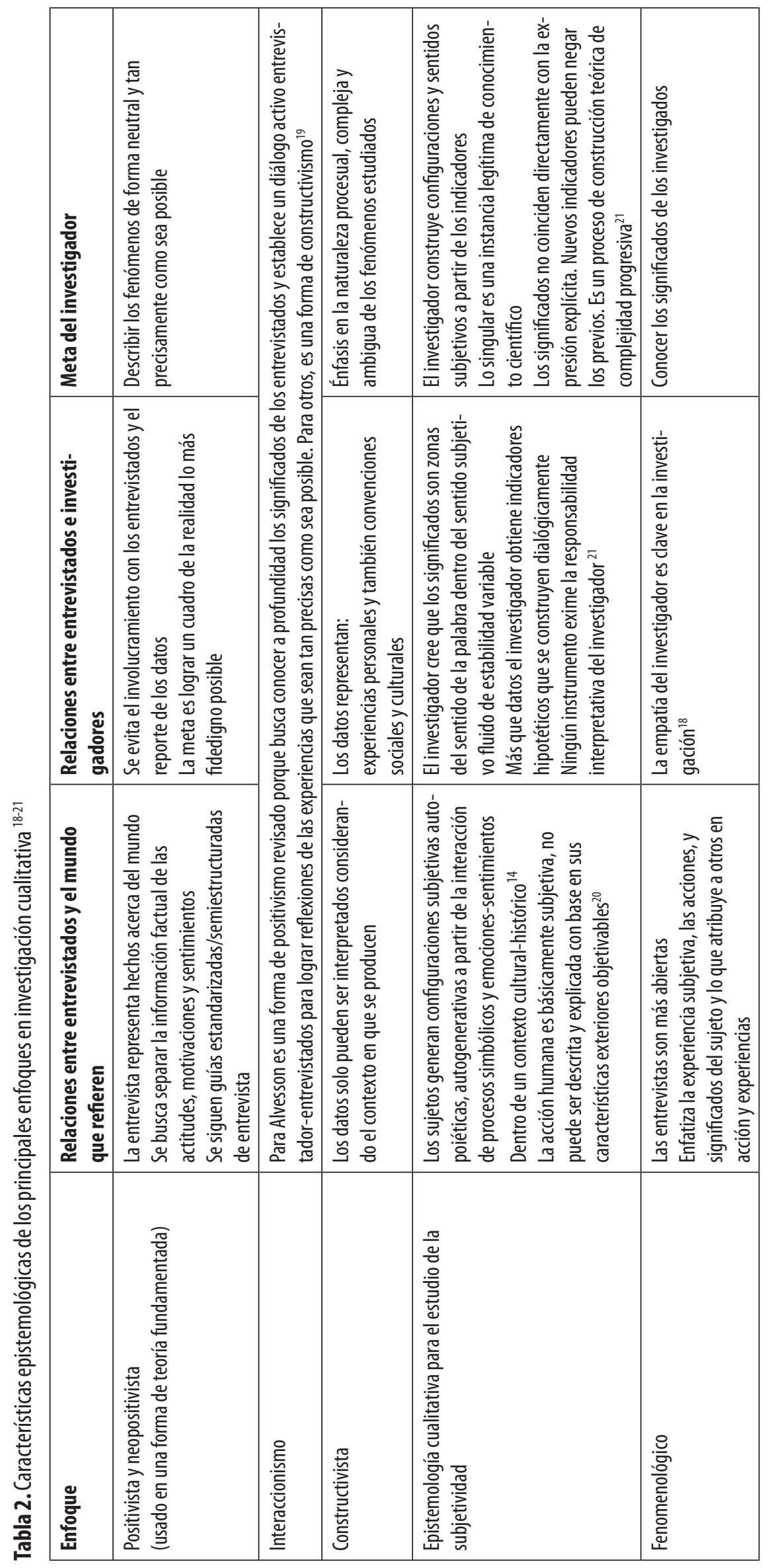


un servicio de mensajería telefónica siendo aceptada sin barreras.

\section{Instrumento de obtención de datos}

Se utilizó una entrevista semiestructurada con las siguientes preguntas:

1. ¿Cuánto tiempo tienes ejerciendo la medicina y cómo ha sido tu experiencia en la relación con tus pacientes?

2. De acuerdo con tu experiencia, ¿crees que la relación médico-paciente puede llegar a tener un efecto terapéutico? ¿sí? ¿no? ¿por qué?

3. De acuerdo con tu experiencia clínica, ¿qué elementos crees que son indispensables para construir una relación médico-paciente con poder terapéutico?

4. ¿Crees que los elementos que acabas de mencionar pueden enseñarse a estudiantes de medicina y residentes de medicina familiar?

\section{Procedimiento de obtención de datos y aspectos éticos}

Se obtuvo el consentimiento verbal para realizar, grabar, conservar las entrevistas, su calendarización, horario y duración máxima (una hora). Las entrevistas se hicieron por videollamada por el sistema Zoom gratuito y fueron grabadas previo consentimiento verbal y compromiso de confidencialidad y privacidad de lo expresado por los participantes. Se mantuvo el compromiso de que solo el investigador tendría acceso a los registros originales, y que las transcripciones omiten identificadores directos y en lo posible los indirectos. Hubo acuerdo de los entrevistados.

Las entrevistas fueron transcritas palabra por palabra utilizando la versión grabada en audio y el servicio en línea Otranscribe.

\section{Sistematización y análisis}

La mayoría de los códigos fueron obtenidos a partir de la lectura de la transcripción y algunos de apuntes en el momento de las entrevistas o inmediatamente después de las notas de campo, en la primera ronda se obtuvieron 32 códigos, con 10 memos. Se realizó un proceso comparativo constante entre los códigos de ambas entrevistas. En la segunda y tercera ronda de codificación después de la retroalimentación discutida en el curso de investigación cualitativa II de un doctorado en investigación psicológica, los códigos se redujeron a 27 y se hicieron más cortos. Los códigos fueron agrupados en seis categorías o temas: comunicación, empatía, enseñanza difícil, recursos de enseñanza, relación médico-paciente terapéutica, códigos del investigador. Los memos se agruparon al final en dos grupos: enseñanza pros y contras y elementos terapéuticos de la relación médico-paciente. La arquitectura del estudio se describe en la figura 1.

El proceso de sistematización se inició de forma manual, pero se logró trasladar al sistema analítico del software Atlas.ti. Se imprimieron informes por códigos agrupados (seis) y los dos agrupamientos de memos a los que se logró ligar con citas registradas codificadas. Fue de gran ayuda la impresión del libro de códigos, que incluía, además de su definición, las líneas iniciales de citas, los grupos a los que pertenecen los códigos y los códigos con que se relacionan más la tipificación de su relación. Los impresos descritos permitieron llegar eficientemente hasta la cita completa en los documentos transcritos de las entrevistas y las notas de campo. La arquitectura del estudio se describe en la figura 1 . Se anexa la tabla 3, de códigos, como ejemplo del proceso seguido desde la codificación de la transcripción hasta la formación de memos en la segunda ronda de análisis. La lista completa es alrededor de siete páginas, más las producidas por el programa Atlas.ti.

\section{RESULTADOS}

Una primera vista de las citas aportadas por cada entrevista muestra que la entrevista 1 produjo el $80 \%$ de las citas del grupo de códigos de comunicación y el $94 \%$ en el de dificultades para la enseñanza (Tabla 4). No obstante, la única cita del renglón 


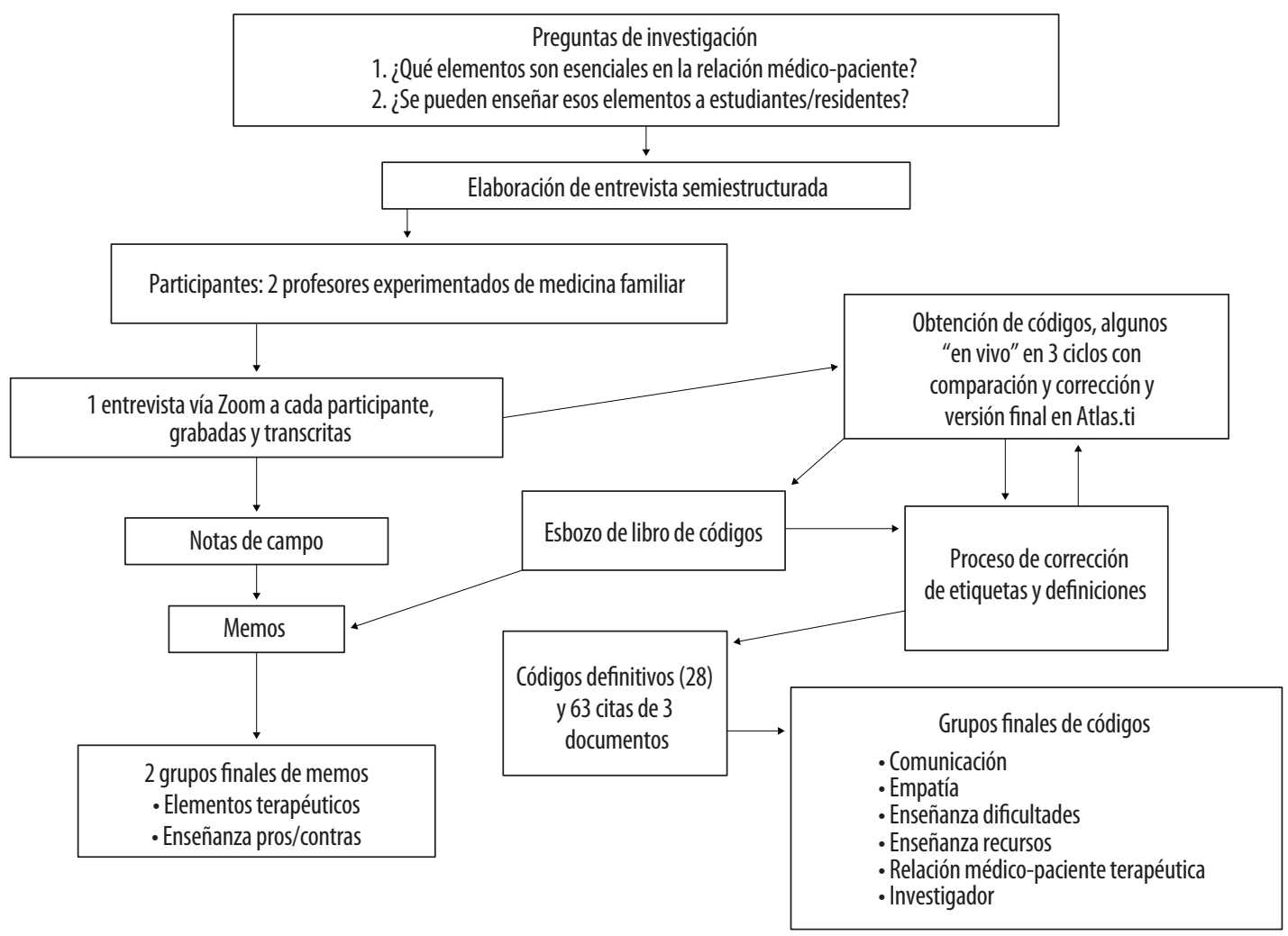

Figura 1. Arquitectura del estudio. Las entrevistas fueron codificadas en tres ciclos haciendo comparación constante en un proceso abierto de corrección de etiquetas de códigos, hubo generación de memos y sus respectivas etiquetas en cada ciclo, hasta la formación final de categorías (códigos agrupados) y memos finales.

de dificultades para la enseñanza de la entrevista 2 es particularmente trascendente, porque afirma que la educación que recibió en la residencia de medicina familiar no le facultó para ejercer profesionalmente:

«Nada de lo que vi en la residencia de medicina familiar me preparó para la experiencia que es ejercer como médico... puede uno ejercer como médico familiar pero infeliz...»

La frase se codificó como «enseñanza fallida».

El resto de las citas parecen estar distribuidas de manera equitativa entre las entrevistas (Tabla 4). Una interpretación de las diferencias podría ser que el entrevistado 1 estuviera más inclinado a hablar de las dificultades en la enseñanza de la relación terapéutica médico-paciente, mientras la otra entrevistada se enfocó más en los medios que facilitan tal enseñanza (Tabla 4).
A continuación se exponen los resultados por grupos de códigos en conexión con las preguntas de investigación estudiadas, no confundir con las preguntas del instrumento usado para responder las preguntas de investigación.

\section{Pregunta de investigación 1}

¿Cuáles serían los elementos terapéuticos de la relación médico-paciente? (Los grupos de códigos fueron construidos con citas de las cuatro preguntas de la entrevistas.)

\section{Grupo comunicación}

Los códigos maduración-ascenso, relación difícil y comunicar amor muestran que establecer una comunicación adecuada con los pacientes es un proceso que tomó años a los participantes:

«Tenemos que conocernos muy bien como personas, como médicos, porque todas 
Tabla 3. Ejemplos del proceso de creación de etiquetas cortas (códigos) a partir de frases, oraciones o palabras de las transcripciones de las entrevistas, y sus respectivos memos en diversos ciclos*

\begin{tabular}{|c|c|c|}
\hline (código) Etiqueta & Ejemplo(s) & Memos \\
\hline Ascenso & «Creo que fue ascendente» & \\
\hline Autoconocimiento & $\begin{array}{l}\text { «Tenemos que conocernos muy bien } \\
\text { como personas ... porque todas nues- } \\
\text { tras emociones pueden intervenir en } \\
\text { nuestra comunicación con el paciente» }\end{array}$ & $\begin{array}{l}\text { El } 26 \text { de abril me doy cuenta de que el autoconocimiento no debe } \\
\text { limitarse a la percepción de emociones. Es necesario incluir ideas, y } \\
\text { sentimientos más elaborados }\end{array}$ \\
\hline Barrera abstracción & $\begin{array}{l}\text { «¿Que importa más? Pues ver el } \\
\text { ecosonograma, la radiografía, el } \\
\text { estudio de laboratorio, las enzimas... } \\
\text { y los muchachos lo que quieren } \\
\text { aprender...» }\end{array}$ & $\begin{array}{l}\text { Memo orientado por teoría: } \\
\text { Conforme se recurre a más estudios de imagen o laboratorio se } \\
\text { eleva el nivel de abstracción y más lejos queda la persona real. La } \\
\text { atención se concentra en una imagen digital o una cifra de biomar- } \\
\text { cadores. Se profundiza sobre la enfermedad hasta abstraerla de la } \\
\text { esencia de la persona. Es un subcódigo de dificultad para enseñar } \\
\text { empatía/humanismo }\end{array}$ \\
\hline Beneficio muto & $\begin{array}{l}\text { «Doctor, me salvó la } \\
\text { vida... la señora me vio y dijo... el } \\
\text { doctor es mi salvador. Y la abuelita de } \\
\text { mi amigo Rafael dice, sí, es muy buen } \\
\text { doctor... yo me sentí muy bien» }\end{array}$ & $\begin{array}{l}\text { Es reconocido en la literatura que el agradecimiento de los pacien- } \\
\text { tes constituye una fuente de salud afectiva de los clínicos }\end{array}$ \\
\hline $\begin{array}{l}\text { Compasión nutridora } \\
\text { bilateral }\end{array}$ & $\begin{array}{l}\text { «He desarrollado tanta compasión } \\
\text { hacia ellos que mi ejercicio ha sido } \\
\text { nutritivo, les ha ayudado a ellos y } \\
\text { también me ha ayudado mucho a mí» }\end{array}$ & $\begin{array}{l}2 .^{\circ} \text { ciclo } \\
\text { El énfasis en los beneficios mutuos de la compasión tiene apoyo } \\
\text { teórico cuando se une a ecuanimidad }\end{array}$ \\
\hline Comunicar amor & $\begin{array}{l}\text { «¿Sabe que está ahí su esposo? Muy } \\
\text { triste, ya va a llorar porque usted está } \\
\text { enferma... ¿Es cierto doctor? Sí es } \\
\text { cierto...., está su esposo, allá afuera, } \\
\text { muy triste ... dice: Déjelo pasar. } \\
\text { Entró el señor y al poco rato se le quitó } \\
\text { el dolor (a la paciente)» }\end{array}$ & $\begin{array}{l}\text { Me parece que hay una intuición humana que comunicar el } \\
\text { amor de un ser cercano a alguien desvalido tiene algún efecto } \\
\text { terapéutico }\end{array}$ \\
\hline
\end{tabular}

* Los códigos fueron surgiendo sin teoría previa, y al mismo tiempo se definieron en el libro de códigos desde el inicio. El libro de códigos fue modificado conforme surgían nuevos elementos. Algunos códigos se eliminaron, otros se fusionaron en las fases 2 y 3 del análisis.

nuestras emociones pueden intervenir en nuestra comunicación con el paciente».

"Ahora me siento mucho mejor, creo que entre más tiempo pasa me siento mejor en el trato con los pacientes».

Y que el proceso de comunicación es más difícil cuando hay diferencias culturales:

«Sentí dificultad para comunicarme con ellos, ¡es la verdad! Era gente de pueblo, yo era de ciudad».

El código relación difícil tuvo su contraparte, comunicar amor, que aparece ligado a la recuperación de una paciente cuando el médico le comunica que su esposo está extremadamente triste y preocupado por ella:
«... el decirle que el marido la amaba. Pues le dio mucha, mucha, vida a la señora, se sintió con muchos deseos de vivir».

\section{Grupo empatía}

Incluye cuatro códigos: compasión nutridora bilateral, empatía-desarrollo, empatía-semilla, empatía-humanismo. El primer código recoge la idea de que la compasión puede ser benéfica para ambas partes en la relación médico-paciente:

La empatía en el ámbito de la relación médico-paciente no parece ser algo dado, sino un desarrollo que parte de una cierta «siembra» que surge de la enseñanza/ 
Tabla 4. Códigos y citas aportados por cada entrevista

\begin{tabular}{|l|c|c|c|c|c|}
\hline \multirow{2}{*}{} & \multicolumn{2}{|c|}{ Entrevista 2 } & \multicolumn{2}{c|}{ Entrevista 1 } & Totales \\
\cline { 2 - 6 } & Absoluto & Relativo de fila & Absoluto & Relativo de fila & Absoluto \\
\hline Comunicación & 2 & $2 / 10$ & 8 & $8 / 10$ & 10 \\
\hline Empatía & 3 & $3 / 6$ & 3 & $3 / 6$ & 6 \\
\hline Enseñanza difícil & 1 & $1 / 16$ & 15 & $15 / 16$ & 16 \\
\hline Enseñanza recursos & 7 & $7 / 15$ & 8 & $8 / 15$ & 15 \\
\hline Relación m-p terapéutica & 5 & $5 / 11$ & 6 & $6 / 11$ & 11 \\
\hline Totales & 18 & & 40 & & 58 \\
\hline
\end{tabular}

experiencia de ver al otro como semejante y verse a sí mismo como un semejante:

Los códigos de empatía en este estudio están ligados a la confianza mutua:

«Lo primero es la confianza, ellos confían en mí y yo en ellos»; la confianza generada por la empatía aparentemente permite tomar mejores decisiones:

«Eso de generar confianza a mí me da mucha tranquilidad, porque estamos tanto el paciente como yo tomando las mejores decisiones».

\section{Grupo relación médico-paciente terapéutica}

El grupo incluye siete códigos: comunicar amor, confianza mutua, efecto terapéutico, autoconocimiento, beneficio mutuo, genuino interés y responsabilidad limitada; los primeros dos ya han sido descritos.

El autoconocimiento se refiere a las expresiones que denotan reflexión sobre los propios sentimientos, emociones y pensamientos que ocurren en la relación médico-paciente; el autoconocimiento se vincula con comunicar amor a los pacientes de forma adecuada:

«Tenemos que conocernos muy bien como personas, como médicos, porque todas nuestras emociones pueden intervenir en nuestra comunicación con el paciente».

El beneficio mutuo está vinculado al agradecimiento de los pacientes que han evolucionado favorablemente:

«Dos meses después, la señora me dijo "el doctor me salvó la vida. El doctor es mi salvador". Y la abuelita de mi amigo Rafael indicó: "sí, es muy buen doctor. Yo me sentí muy bien"».

El interés genuino representa expresiones donde se destaca la relación que tiene con el resultado terapéutico:

«Las personas mejoran con el solo hecho de tener un observador que esté pendiente de ellos, y que esté interesado genuinamente en ellos».

El código de responsabilidad limitada recoge expresiones de los entrevistados que reconocen la necesidad de algún limite en las responsabilidades del médico respecto a su paciente, no se puede ni se debe intentar hacerse cargo por completo de sus dificultades de vida.

«... ser claros, ser directos, poner límites, porque, aunque uno quiera verlos como familia hay ocasiones en las que se deben poner límites... para que podamos desarrollarnos como personas en otras áreas».

\section{Pregunta de investigación 2}

¿Es posible enseñar a los estudiantes/residentes los elementos terapéuticos esbozados aquí?

Los datos que podrían ayudar a responder esta pregunta de investigación fueron reunidos en dos grupos de códigos: dificultades y recursos de la enseñanza de los elementos terapéuticos detectados, cada uno con siete códigos. La primera dificultad referida por los entrevistados es que el enfoque humanista de la relación médico-paciente no tiene lugar formal en el currículo médico: 
«... es difícil porque no está del todo en el programa la experiencia que tengo en la universidad... hay un programa y contenidos rígidos y hay que seguirlo...».

La complejidad de la enseñanza afectiva se complica con el deseo de los estudiantes de aprender técnicas biomédicas cada vez más complejas y numerosas que responden a una educación que prioriza la abstracción de la enfermedad representada en imágenes computarizadas y biomarcadores; la situación se acentúa en los ambientes del hospital:

«¿Que importa más? ... ver el ecosonograma, la radiografía, el estudio de laboratorio, las enzimas y los muchachos lo que quieren es aprender, más que la persona».

En esta última frase está implícito que los roles que se observan en los tutores pueden ser un antiejemplo de empatía y humanismo. También se mencionó el ambiente social global orientado a la eficiencia e ignorando el lado humano y priorizando resultados inmediatos, como un factor que dificulta la enseñanza humanista.

«La sociedad misma impide, dificulta, el humanismo... a resolver las cosas con menos esfuerzo y tener los resultados rápido y bien... entonces nos olvidamos de la gente».

Estas dificultades describen un ambiente muy desfavorable para la educación empática del médico de todas las especialidades, pero es especialmente deteriorante de la labor del médico familiar cuyo poder terapéutico fundamental subyace en su personalidad, como describió Michael Balint en $1960^{1}$. Por eso la relevancia del dato aportado por un participante:

«Nada de lo que vi en la residencia de medicina familiar me preparó para la experiencia de ejercer como médico, puede uno ejercer como médico familiar pero infeliz...».

Las dificultades descritas se equilibran con las aportaciones de recursos encontrados en estas dos entrevistas. Un entrevistado explica que su reflexión personal de casos que le movieron intensas emociones, tanto negativas como positivas, le permitieron lograr avances importantes en su proceso de autoconocimiento: «Una de las habilidades que desarrollé en la práctica, fue identificar qué paciente me da algo negativo o algo muy positivo, porque a partir de ahí pude ampliar esa manera de ver mis propias emociones... aprovechar todos esos casos que nos hacen la vida difícil y desarrollarnos en la construcción de la relación con el paciente».

Los entrevistados también describen el beneficio de acercamientos teóricos que les permitieron avanzar en el manejo adecuado de sus emociones:

«...aprendí todavía más de la teoría (de Balint) de la relación médico paciente; ... pues sí, es mejor trabajarlo (las emociones) para identificar como ser humano qué es lo que me está pasando».

Uno de los entrevistados reconoció haber pasado por una fase que describió como "crueldad e ignorancia":

"Yo era tonto, recién salido de la residencia. Mi ignorancia, ¡muy grande! En urgencias le drené el absceso anal y el pobre hombre se quejaba mucho. Al salir dijo "jestos son unos salvajes!". Sí, sentí que estaba con mucho dolor. Cuando leí cómo se debía drenar un absceso con anestesia, dije "iqué idiota, pobre hombre! ¡Cómo lo hice sufrir! ¡Mi ignorancia"».

\section{DISCUSIÓN}

¿Qué parecen decir los datos? Parece que es razonable inferir que ambos entrevistados coinciden en que la relación médico-paciente puede tener efectos terapéuticos; y que, para ello, la relación debe contener componentes o elementos complejos, tales como capacidad empática comprendida como sentir la humanidad del otro, y capacidad para sentir compasión (compartir el sufrimiento) ${ }^{13}$ que podría nutrir a ambas partes de la relación. Tales capacidades empáticas podrían ser vistas con una metáfora botánica, una semilla que se planta en la infancia o que también puede desarrollarse en la edad adulta cuando se percibe, se siente, se comparte el sufrimiento del otro. Y que 
la combinación de cierta práctica clínica reflexiva y teoría propicia es capaz de hacer florecer las capacidades compasivas. Los datos parecen apoyar la idea de que la reflexión sobre casos reales que incitan emociones intensas de los médicos pueden ser una vía para desarrollar habilidades de autoconocimiento, justamente la esencia de los grupos Balint ${ }^{22}$; coincide también con las observaciones de Marilyn Fleer con niños que participan en situaciones autodirigidas donde ocurren situaciones emocionalmente cargadas. Los niños avanzan en sus procesos de desarrollo emocional cuando reflexionan sobre lo que experimentaron ${ }^{23}$.

Los datos aportan también la idea de la responsabilidad limitada, un elemento equilibrador de la compasión desmedida, una idea que puede ser fácilmente conectada con la ecuanimidad compasiva ${ }^{3,24}$. Otra aportación relevante es que se destacan el efecto negativo de factores culturales utilitaristas en la educación médica y en el conjunto de la sociedad. De manera destacada, se señala al ambiente del hospital como fomentador de relaciones distantes, incluso crueles, dato que coincide con la literatura ${ }^{25}$. Y dado que los estudiantes y residentes de medicina familiar deben permanecer por años en el ambiente del hospital, esto representa una gran barrera formativa para la formación en compasión ecuánime. En el mismo sentido está la creciente tecnificación médica ${ }^{26}$.

\section{DEBILIDADESY LIMITACIONES DEL ESTUDIO}

Los entrevistados son solo dos personas y tienen un perfil académico cercano al del investigador, lo que podría significar puntos ciegos. Para limitar estos sesgos, se incluyó una «confesión del investigador» y la codificación de las notas de campo en categoría específica. Se muestran algunas citas relevantes:

«El "testimonio" del profesor X es como una vida condensada que merece ser guardada y mostrada a generaciones jóvenes ».

Para investigar verdaderamente un fenómeno es indispensable que el investigador se sienta realmente perplejo, desconcertado con lo que ve, una "postura científica planteada por Galileo» ${ }^{27}$. El lector deberá ser prevenido de los sesgos señalados y otros no visibles.

\section{CONCLUSIONES PRELIMINARES}

Los resultados descritos generan hipótesis que podrían abrir líneas de investigación con métodos cuantitativos, cualitativos y mixtos, por ejemplo: ¿Piensan los médicos familiares mexicanos que la residencia de medicina familiar los facultó para formar relaciones terapéuticas y enfrentar los complejos casos que atienden? ¿Su paso por el hospital les influyó en algún sentido respecto a su capacidad terapéutica como médico-medicamento? etc. Podemos enumerar las conclusiones de este estudio de la siguiente manera:

1. La relación médico-paciente puede tener efectos terapéuticos y los elementos relacionados deberían enseñarse en el currículo médico formal.

2. Es fundamental la capacidad empática para compartir estados emocionales de los pacientes y saber responder de manera constructiva.

3. El desarrollo empático puede ser visto como una siembra que florece, es un proceso dinámico y único en cada clínico.

4. El desarrollo de autoconocimiento es crucial, y la reflexión sobre los «casos difíciles» podría ser una buena vía para trabajarlo.

5. El ambiente humano y centrado en la tecnología del hospital podría ser deletéreo para el desarrollo de la personalidad terapéutica del residente de medicina familiar.

6. Las residencias de medicina familiar en México podrían estar formando residentes sin las experiencias, herramientas y capacidades para un ejercicio complejo y que les permita «una práctica feliz».

7. Enseñar a establecer límites emocionales en las responsabilidades del médico familiar podría ser conectado con el desarrollo de la ecuanimidad unida a la compasión indispensable; proyecto en el que se inscribe esta investigación exploratoria. 


\section{AGRADECIMIENTOS}

A la Dra. en Psicología Laura Rebeca Mejía Arauz, la Dra. en Comunicación Teresita Morfín López y el Dr. en Educación Antonio Ray Bazán por sus inapreciables aportaciones al diseño y análisis de este trabajo.

\section{FINANCIAMIENTO}

Se recibió la beca \#785445 del Consejo Nacional de Ciencia y Tecnología (CONACYT) de agosto de 2020 a septiembre de 2021 .

\section{CONFLICTO DE INTERESES}

El autor declara no tener conflicto de intereses.

\section{RESPONSABILIDADES ÉTICAS}

Protección de personas y animales. Los autores declaran que para esta investigación no se han realizado experimentos en seres humanos ni en animales.

Confidencialidad de los datos. Los autores declaran que en este artículo no aparecen datos de pacientes.

Derecho a la privacidad y consentimiento informado. Los autores declaran que en este artículo no aparecen datos de pacientes.

\section{BIBLIOGRAFÍA}

1. Balint AJ. Introduction. En: Balint M, editor. The doctor, his patient, and the illness. Edimburgo: Churchill Livingstone; 2000. pp. 1-4.

2. McWhinney IR. Being a general practitioner: What it means. Eur J Gen Pract. 2000;6(December):135-9.

3. Adler HM. The sociophysiology of caring in the doctor-patient relationship. J Gen Intern Med. 2002;17(11):883-90.

4. Halpern J. What is clinical empathy. J Gen Intern Med. 2003;18(August):670-4.

5. Benito-Oliver E, Rivera-Rivera P. El cultivo de la autoconciencia y el bienestar emocional en los profesionales que trabajan con el sufrimiento. Rev Inv Educ Ciencias Salud. 2019;4(S1):77-92.
6. Olaisen RH, Schluchter MD, Flock SA, Koroukian SM, Stange KC. Assessing the longitudinal impact of physician patient relationship on functional health. Ann Fam Med. 2020;18(5):422-9.

7. Scott JG, Cohen D, Dicicco B, Miller WL, Stange K. Understanding healing relationships in primary care. Ann Fam Med. 2008;6(4):315-22.

8. Roth-Damas $P$, Portillo-Santamaria M, Folch-Marín B, PalopLarrea V. Fibromialgia y empatía: Un camino hacia el bienestar. Atención Primaria. 2017;50(1):69-70.

9. Ramírez-Villaseñor I. Sentimientos del estudiante de medicina al abordar la muerte con cine, música y casos reales. Educ Med (España). 2019;20(5):267-71.

10. Ramírez-Villaseñor I. Emociones, relación médico-paciente y cine: Comparación entre estudiantes y médicos familiares. Una investigación cualitativa. Rev Mex Med Fam. 2016;3(2):15-23.

11. US National Library of Medicine. Medical Subject Headings [Internet]. National Institutes of Health (NIH) [consultado: $11 \mathrm{de}$ noviembre de 2021]. Disponible en: https://www.nlm.nih.gov/ mesh/meshhome.html

12. Lederman W. A. Andes Pediatr. 2007;78(5):459-60

13. Post SG, Ng LE, Fischel JE, Bennett M, Bily L. Routine, empathic and compassionate patient care: definitions, development, obstacles, education, and beneficiaries. J Eval Clin Pract. 2014;20(6):872-80.

14. González-Rey F. La subjetividad en una perspectiva cultural-histórica: avanzando sobre un legado inconcluso. CS. 2013;2(1):19-42.

15. Pembroke N. Empathic and compassionate healthcare as a Christian spiritual practice. Practical Theology. 2019;12(2):133-46.

16. Charmaz K. Reconstructing theorizing in grounded theory studies. In: Charmaz K (ed.) Constructing grounded theory (Introducing qualitative methods series). Los Angeles, Ca: SAGE Publication; 2014:225-260.

17. Flick U. The qualitative research process Sage. 5th ed. Londres: Sage; 2014.

18. Jarvinen M, Mik-Meyer N. Qualitative analysis: Eight approaches for the social sciences. Los Angeles: SAGE Publications; 2020.

19. Alvesson M. Interpreting Interviews. London: Sage Publication 2011.

20. Sousa-Santos B. Un discurso sobre las ciencias. In: Meneses PA, Arriscado-Nunes C (eds.) Boaventura De Sousa Santos: Construyendo las epistemologías del sur. Buenos Aires: CLACSO 2018:31-72.

21. González-Rey F, Mitjáns-Martínez A. Una epistemología para el estudio de la subjetividad: Sus implicaciones metodológicas. Psicoperspectivas. 2016;15(1):5-16.

22. Balint $M$. The structure of the training-cum-research-seminars. Its implications for medicine. J Roy Coll Gen Practit. 1969;17(81):201-11.

23. Fleer $M$. Foregrounding emotional imagination in every day preschool practices to support emotion regulation. En González-Rey F, Veresov N, (ed.) Perezhivanie, emotions and subjectivity: Advancing Vygotsky's legacy Perspectives in cultural historical research. Singapur: Springer; 2017:85-104.

24. Adler HM. The history of the present illness as treatment: who's listening and why does it matter? J Am Board Fam Pract. 1997; 10(1):28-35.

25. Berwick DM. What 'patient-centered' should mean: Confessions of an extremist. Health Affairs. 2009:28(4):w555-w556.

26. Miles A. Science, humanism, judgement, ethics: Personcentered medicine as an emergent model of modern clinical practice. Folia Med (Plovdiv). 2013;55(1):5-24.

27. Chomsky N. ¿Qué clase de criaturas somos? 1a. edición. Ciudad de México: Ediciones Culturales Paidós; 2017. 